\title{
Hierarchical TS-1 zeolite for the oxidation of bulky molecules
}

\author{
A. Silvestre-Albero ${ }^{1}$, A. Grau-Atienza ${ }^{2}$, E. Serrano ${ }^{2}$, J. García-Martínez $^{2, *}$ and J. Silvestre- \\ Albero $^{1, *}$ \\ ${ }^{1}$ Laboratorio de Materiales Avanzados, Departamento de Química Inorgánica-Instituto \\ Universitario de Materiales, Universidad de Alicante, Ap. 99, E-03080 Alicante (Spain). \\ ${ }^{2}$ Laboratorio de Nanotecnología Molecular, Departamento de Química Inorgánica, \\ Universidad de Alicante, Ap. 99, E-03080 Alicante (Spain).
}

\section{Highlights}

Desilication of TS-1 using $\mathrm{NaOH}$ gives rise to hierarchical zeolites.

$>$ Desilicated TS-1 exhibits a large BET surface area together with a well-developed mesoporosity.

Hierarchical TS-1 remains the activity of the original TS-1 for oxidation of small molecule.

Hierarchical TS- 1 shows a significant improvement compared to original TS- 1 for the oxidation of bulky molecules.

Corresponding authors:

Joaquín Silvestre-Albero

Tel.: +34965909350 ext. 2226

Fax: +34965903454

Email: joaquin.silvestre@ua.es

Javier García-Martínez

Tel.: +34965909350 ext. 2372

Fax: +34965903454

Email: j.garcia@ua.es 


\begin{abstract}
A series of hierarchical TS-1 zeolites have been produced by desilication of the original TS-1 (4 wt.\% Ti) using a chemical treatment with $\mathrm{NaOH}$. Desilicated TS-1 zeolites exhibit a large BET surface area together with a well-developed mesoporosity. The hierarchical samples show good catalytic activity for the oxidation of small molecules while significantly higher activity for the oxidation of bulky molecules.
\end{abstract}

Keywords: Desilication, hierarchical zeolites, TS-1, epoxidation reactions 


\section{Introduction}

Since the discovery of titanium-silicalite (TS-1) by Taramasso in 1983 [1], this zeolite has been widely applied to numerous oxidation reactions including aromatic hydroxylation and alkene epoxidation using mild conditions [2,3]. Besides the high catalytic activity shown by isolated framework titanium species in TS-1 -mainly tetrahedrally coordinated titanium-, this zeolite is inefficient for the selective oxidation of bulky molecules due to its narrow pore structure (pore entrance of ca. 0.54).

In order to minimize diffusional limitations in the microporous network of MFI zeolites, several approaches have been developed in the last few years to induce intra-crystalline mesoporosity including hard templating [4-6], soft templating [7,8], demetalation [9-15] and modified crystallization methods $[16,17]$. Unfortunately, several of these approaches provide structures which are not hydrothermally stable in a manner similar to nanocrystalline zeolites. Concerning demetalation post-treatment methods, Groen et al. reported that ZSM-5 zeolite with a Si/Al ratio of $\sim 25-50$ can be conveniently desilicated by alkaline treatment, thus producing mesopores which enhance the diffusional properties of this material [10]. Despite the many articles devoted to the synthesis, characterization and application of desilicated ZSM-5, the studies on hierarchical TS-1 prepared using the desilication approach are much more limited [14,15].

Herein we report the preparation of hierarchical TS- 1 by desilication with NaOH. TS-1 is a titanium-silicate which shares the same crystalline structure with ZSM-5, namely MFI. The catalytic performance of the former is due by the presence of tetrahedrally coordinated $\mathrm{Ti}$ atoms in the framework. As previously reported [9-19], silicon is preferentially extracted into solution upon alkaline treatment while $\mathrm{Al}$ in ZSM-5 or Ti in the case of TS-1 remains in the solid. Along this manuscript, we address the effect of different alkaline treatments on the physical properties (porosity and crystallinity) and the 
catalytic behavior of desilicated TS-1 zeolites in the oxidation of small and bulky molecules.

\section{Experimental Section}

\subsection{Synthesis of TS-1}

A reported synthesis of TS- 1 type zeolite [19] was judiciously adapted for the synthesis of a TS-1 titanosilicalite with 4 wt.\% titania. In a typical run, $7.5 \mathrm{ml}$ of TPAOH (tetrapropylammoniumhydroxide, $1.0 \mathrm{M}$ aqueous solution, Aldrich) were mixed with 10.5 $\mathrm{ml}$ of distilled water. Following, $300 \mu \mathrm{l}$ of TBOT (titaniumbutoxide 99\%, Acros) and 5.6 $\mathrm{ml}$ of TEOS (tetraethylorthosilicate, 98\%, Acros) were added. The molar composition of the synthesis gel was 1 TBOT: 30 TEOS: 8 TPAOH: $1050 \mathrm{H}_{2} \mathrm{O}$. The final mixture was then reacted at room temperature for $1.5 \mathrm{~h}$ under stirring, followed by hydrothermal aging at $70^{\circ} \mathrm{C}$ for 3 days in a round-bottom flask of $250 \mathrm{ml}$ which equipped with a condenser and placed in an oil bath. Finally, an additional hydrothermal treatment was performed at $150^{\circ} \mathrm{C}$ for 7 days in a Teflon lined stainless steel autoclave $(100 \mathrm{ml})$. Upon cooling at room temperature, the solid product was centrifugated, thoroughly washed, dried overnight and finally calcined at $550^{\circ} \mathrm{C}$ for $6 \mathrm{~h}$.

Post-synthetic treatment of desilication was made following the methodology reported by Groen et al. [10]. The as-synthesized TS-1 sample was treated with different concentrations of $\mathrm{NaOH}$ solution $(0.4,0.6$ and $0.8 \mathrm{M})$ and subsequently with $\mathrm{HCl}$ in order to obtain the three mesoporous samples. In a typical run, $3.3 \mathrm{~g}$ of the as-synthesized TS-1 titanosilicalite was magnetically stirred $(600 \mathrm{rpm})$ in $100 \mathrm{ml}$ of aqueous solution of $\mathrm{NaOH}$ at $65^{\circ} \mathrm{C} 30 \mathrm{~min}$; after that, the solution was filtered off and throughly washed with distilled water. Following, $1 \mathrm{~g}$ of each sample was magnetically stirred (600 rpm) in 100 $\mathrm{ml}$ of $0.1 \mathrm{M}$ aqueous $\mathrm{HCl}$ at $65^{\circ} \mathrm{C}$ during $6 \mathrm{~h}$. Afterwards, the solution was filtered and 
washed with distilled water. Treatment yields vary between 14 and 19\%. Samples were denoted TS-1- $x \mathrm{NaOH}-\mathrm{HCl}$, where $x$ refers to the $\mathrm{NaOH}$ molar concentration used during the treatment.

\subsection{Sample characterization}

The elemental composition of all samples was determined by micro X-ray fluorescence in different regions of the sample. A minimum of three scans per sample was carried out in a Orbis Micro-XRF Analyzer from EDAX. The system includes dual high and low magnification CCD cameras with auto-focus for easy sample positioning and rapid set-up of automated experiments, as well as a large area $\mathrm{Si}(\mathrm{Li})$ detector for best sensitivity.

The morphology of the mesoporous materials was examined by transmission electron microscopy, TEM (JEM-2010 microscope, JEOL, 200kV, $0.14 \mathrm{~nm}$ of resolution). Samples for TEM studies were prepared by dipping a sonicated suspension of the sample (5-10 mg) in ethanol on a carbon/formvar-coated copper grid (300 mesh).

The porous structure of the synthesized materials was analyzed by nitrogen adsorption at $196^{\circ} \mathrm{C}$ using a home-made fully automated equipment designed and constructed by the Advanced Material group (LMA), now commercialized as $\mathrm{N}_{2}$ Gsorb- 6 . The samples were previously degassed $\left(10^{-8} \mathrm{MPa}\right)$ at $150^{\circ} \mathrm{C}$ for $4 \mathrm{~h}$. Textural parameters ("apparent" surface area and micropore volume) were estimated from the nitrogen adsorption data after application of the BET and Dubinin-Radushkevich equation, respectively. The total volume of mesopores was estimated by subtracting the micropore volume to the total volume measured at $\mathrm{P} / \mathrm{P}_{0} \sim 0.95$.

X-ray diffraction (XRD) analysis was carried out with a Bruker D8Advance diffractometer (operating at $40 \mathrm{kV}$ and $40 \mathrm{~mA})$, using a $\mathrm{CuK \alpha}$ radiation $(\lambda=$ $1.54056 \AA)$. The samples were scanned from $5^{\circ}$ to $30^{\circ}(2 \theta)$, at a scanning rate of 
$0.04 \% \mathrm{~min}$. The UV-vis absorbance spectra of the solid as-synthesized samples were collected in a Jasco V-650 spectrophotometer. Spectra were taken in the wavelength range of 200-800 $\mathrm{nm}$ (scan speed $400 \mathrm{~nm} / \mathrm{min}$ and data interval of $1 \mathrm{~nm}$ ).

\subsection{Catalytic measurements}

Catalytic studies were performed using cyclohexene (99\%, Sigma-Aldrich) and 1-tertbutyl-1-cyclohexene ( $80 \%$, Sigma-Aldrich) as substrate and hydrogen peroxide as the primary oxidant. In a typical reaction, $2 \mathrm{mmol}$ of the substrate, $2 \mathrm{mmol}$ of hydrogen peroxide (30 wt. $\%$ in water, Aldrich), $40.0 \mathrm{mg}$ of the catalyst, $0.25 \mathrm{ml}$ of $n$-octane (99\%, Sigma-Aldrich) as internal standard and $5 \mathrm{ml}$ of 1-propanol (99.7\% Sigma-Aldrich) as a solvent up to $5.8 \mathrm{ml}$ final volume were added to a $50 \mathrm{ml}$ round-bottom flask fitted with a reflux condenser. The reaction mixture was stirred at $70^{\circ} \mathrm{C}$ for $2 \mathrm{~h}$. The reaction was terminated by quenching with water. The catalyst was separated by filtration and the reaction products were analyzed by on-line gas-chromatography using a Shimadzu GC2010 equipped with flame ionization detector (FID) and a capillary column HP-5. Blank experiments were performed using the same experimental conditions without catalyst. Before catalytic experiments, samples were dried at $80^{\circ} \mathrm{C}$ in an oven to remove moisture.

\section{Results and discussion}

The morphology of the synthesized samples has been studied by transmission electron microscopy (TEM). Figure 1 displays representative TEM images for the TS- 1 sample before and after the desilication treatment using $\mathrm{NaOH} 0.4 \mathrm{M}$. 

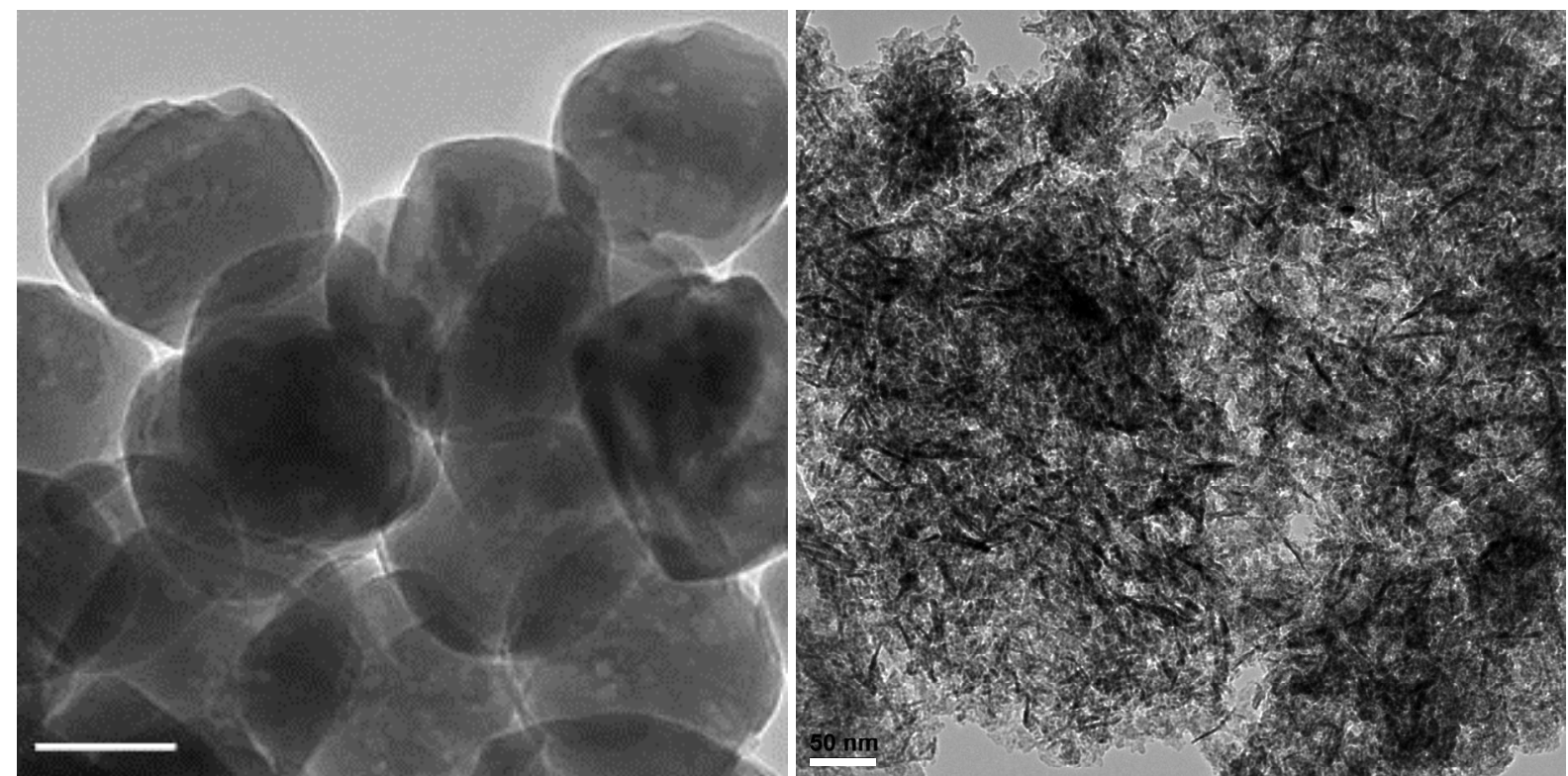

Fig. 1. Representative TEM micrographs of the original TS-1 zeolite (left, scale bar $=100 \mathrm{~nm}$ ) and TS-1 desilicated samples using $\mathrm{NaOH} 0.4 \mathrm{M}$ followed by the $\mathrm{HCl}$ solution post-synthetic treatment (right, scale bar $=50 \mathrm{~nm}$ ).

The as-synthesized TS-1 sample with 4 wt.\% titania exhibits nanocrystals of around 90$120 \mathrm{~nm}$ in diameter, without any visible intracrystalline mesoporosity. In contrast, the desilication treatment with a $\mathrm{NaOH}$ (using concentrations ranging from $0.4 \mathrm{M}$ to $0.8 \mathrm{M}$ ) produces significant modifications of the crystal morphology as can be observed in Figure 1 (right) and Figure S1 in ESI, i.e. preferential formation of intracrystalline mesopores. Elemental composition of samples before and after desilication was determined by micro X-ray fluorescence and listed in Table 1. As expected, the amount of $\mathrm{Ti}$ increases considerably after the different desilication treatments in detriment of the silicon content. This observation clearly suggests that the observed formation of intracrystalline mesoporosity must be attributed to the selective removal of silica from the zeolite framework. At this point it is interesting to highlight that the formation of large cavities or voids in the mesoporous range is highly desirable because these will constitute the pathways for reactant and product molecules in/out of the active sites in a subsequent catalytic application. 
The deterioration of the crystal structure after the desilication treatment is further confirmed by comparing the XRD patterns of the synthesized TS-1 and demetalated samples. The original TS-1 sample (Fig. 2) exhibits the typical XRD pattern of silicalite1. The XRD pattern of the sample desilicated with the mildest conditions $(0.4 \mathrm{M} \mathrm{NaOH})$ also shows peaks characteristics of TS-1 although with a very weak intensity. Interestingly, those peaks totally dissapear for higher $\mathrm{NaOH}$ concentrations, i.e. only a broad peak characteristic of an XRD-amorphous structure can be observed.

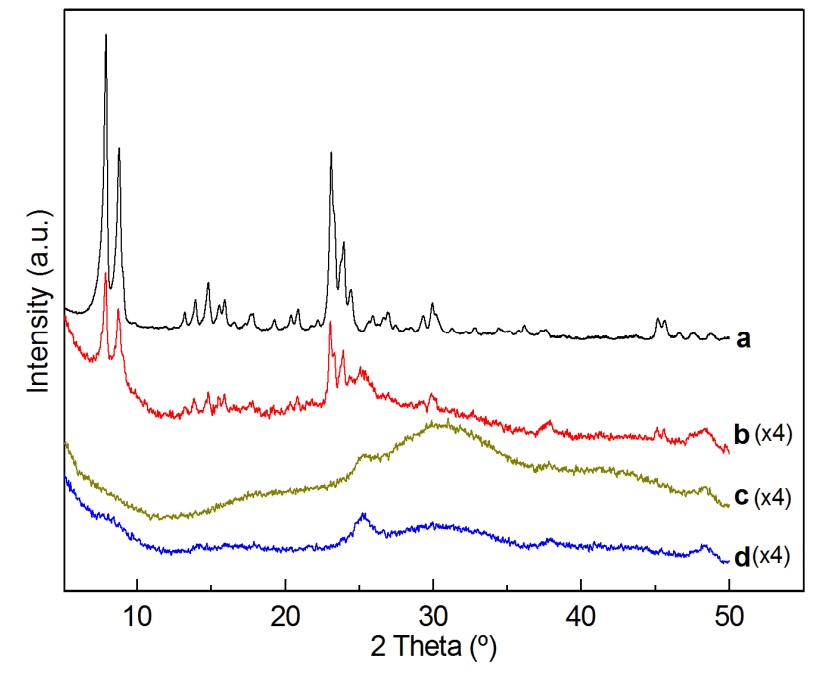

Fig. 2. XRD patterns corresponding to the (a) original TS-1 zeolite and TS- 1 treated with $\mathrm{NaOH}$ (b) $0.4 \mathrm{M}$, (c) $0.6 \mathrm{M}$ and (d) $0.8 \mathrm{M}$, followed by a $0.1 \mathrm{M} \mathrm{HCl}$ washing step. Spectra have been vertically shifted for clarity.

Previous studies have shown that the crystallographic properties of desilicated TS-1 samples can be partially preserved when using $\mathrm{NaOH}$ concentrations lower than those used in this work, c.a. $0.2 \mathrm{M}$, although this mild treatment produces only a slight development of mesoporosity [14]. Consequently, the deterioration observed in our samples must be attributed to the use of highly concentrated solutions, thus giving rise to samples with an extremely low Si/Ti ratio (see Table 1). 


\section{Table 1}

Chemical composition and textural parameters of the synthesized materials.

\begin{tabular}{lccccc}
\hline Sample & $\begin{array}{c}\text { Ti content }^{\mathrm{a}} \\
(\text { wt. \% })\end{array}$ & $\mathrm{Si} / \mathrm{Ti}^{\mathrm{b}}$ & $\begin{array}{c}\mathrm{S}_{\mathrm{BET}}{ }^{\mathrm{c}} \\
\left(\mathrm{m}^{2} \mathrm{~g}^{-1}\right)\end{array}$ & $\begin{array}{c}\mathrm{V}_{\text {micro }} \mathrm{d}^{(} \\
\left(\mathrm{cm}^{3} \mathrm{~g}^{-1}\right)\end{array}$ & $\begin{array}{c}\mathrm{V}_{\text {meso }} \mathrm{e} \\
\left(\mathrm{cm}^{3} \mathrm{~g}^{-1}\right)\end{array}$ \\
\hline TS-1 & 2.6 & 29.0 & 440 & 0.17 & 0.39 \\
TS-1-0.4NaOH-HCl & 21.9 & 2.3 & 185 & 0.07 & 0.73 \\
TS-1-0.6NaOH-HCl & 25.7 & 1.8 & 225 & 0.08 & 0.66 \\
TS-1-0.8NaOH-HCl & 35.1 & 0.9 & 260 & 0.09 & 0.46 \\
\hline
\end{tabular}

${ }^{a}$ Average Ti content and ${ }^{b} \mathrm{Si} / \mathrm{Ti}$ molar ratio determined by micro X-Ray fluorescence. ${ }^{c}$ BET surface area. ${ }^{d}$ Micropore volume calculated from the adsorption branch according to the DubininRadushkevich equation. ${ }^{e}$ Mesopore volume calculated from the difference between the total pore volume at $\mathrm{P} / \mathrm{P}_{0} \sim 1$ and the micropore volume.

To study the changes in the textural properties caused by the different alkaline treatments tested, $\mathrm{N}_{2}$ adsorption-desorption isotherms were performed at $-196^{\circ} \mathrm{C}$ to all the samples. The nitrogen adsorption/desorption isotherms of the as-synthesized materials, as well as the corresponding pore size distribution are shown in Fig. 3. Textural parameters have been calculated from the adsorption data and listed in the Table 1. As shown in Fig. 3, the original TS-1 sample exhibits a type I isotherm (according to the IUPAC classification) with a narrow knee at low relative pressures and relatively low adsorption at intermediate pressures, in close agreement with its microporous structure. The hysteresis loop at high relative pressures $\left(\mathrm{P} / \mathrm{P}_{0}>0.9\right)$ is usually attributed to the condensation within the voids formed between the zeolitic particles [22], thus confirming the small size of the TS-1 crystallites (see Fig. 1, left). The desilication treatment with $\mathrm{NaOH}$ produces important changes in the adsorption isotherms for all samples. The amount of nitrogen adsorbed at low relative pressures drastically decreases, thus confirming the deterioration of the microporous structure after the alkaline treatment. Furthermore, all the desilicated samples show nitrogen adsorption at intermediate-high relative pressures, which can be related to the development of a secondary porosity, i.e. mesoporosity (see Fig. 3, right), which is further confirmed by the increase in the mesopore volume of the desilicated 
samples in comparison with the parent TS-1 zeolite (see Table 1).
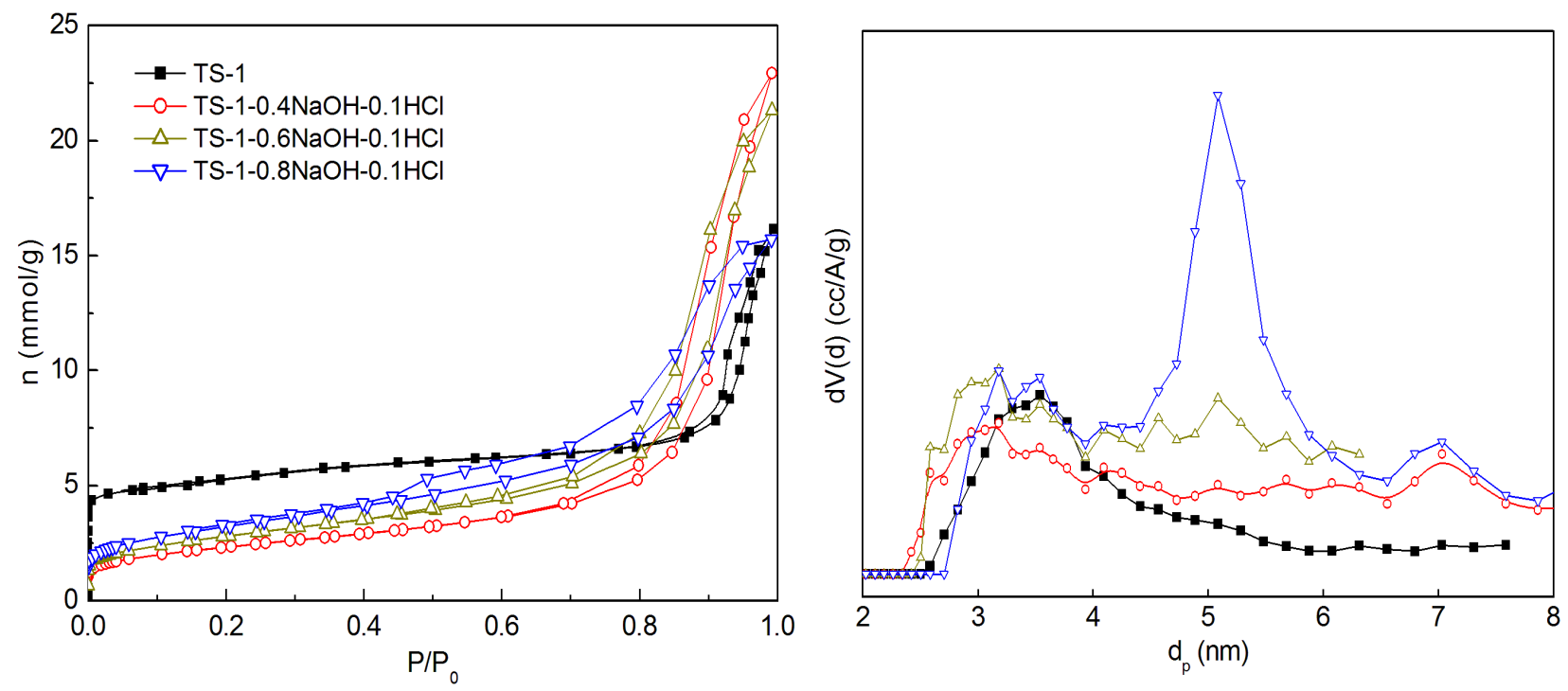

Fig. 3. $\mathrm{N}_{2}$ adsorption-desorption isotherms (left) and the corresponding pore size distributions calculated by applying NL-DTF to the absorption branch (right) for the parent TS-1 and the desilicated samples.

Interestingly, the largest development of mesoporosity is observed for the sample chemically treated with the lower $\mathrm{NaOH}$ concentration $(0.4 \mathrm{M})$ with a total mesopore volume of $0.73 \mathrm{~cm}^{3} \cdot \mathrm{g}^{-1}$. The large nitrogen uptake at $\mathrm{P} / \mathrm{P}_{0}>0.7-0.8$ is accompanied by the presence of a type $\mathrm{H} 4$ hysteresis loop, characteristic of solids containing mesoporosity. An increase in the hardness of the chemical treatment becomes detrimental for the development of mesopores. As can be observed in Table 1, there is a decrease in the mesopore volume for large $\mathrm{NaOH}$ concentrations probably due to the excessive removal of silica from the zeolite network after the chemical treatment, thus giving rise to the deterioration of zeolite network in terms of structure and cristallinity. However, at this point it is noteworthy to highlight that this deterioration, at least on sample TS-1$0.8 \mathrm{NaOH}-0.1 \mathrm{HCl}$, gives rise to a different hysteresis loop (different type of mesopores), which is reflected in the pore size distribution by a contribution at around 4-6 nm (see Fig. 3 right).

Furthermore, these amorphous materials still preserve some microporosity (around 0.09 
cc/g, see Table 1), probably due to the fact that even if long-order cristallinity is lost, some TS-1 fragments still remain after the desilication process. Additionally, the deterioration of the crystal structure is accompanied by an increase in the BET surface area due to the contribution from the newly developed mesopores ( $c a$. 4-6 nm) and also some micropores (slight increase in the nitrogen adsorption capacity at low relative pressures) probably produced by the partial structural collapse of TS-1 units under aggressive alkaline conditions.

Fig. 4 shows the UV-vis spectra of the parent TS-1 and the desilicated samples. Catalytic activity of titanium-containing materials is related to the coordination of the Ti active sites and, more specifically, to the tetrahedrally coordinated Ti(IV) sites, which can be easily analyzed by UV-vis spectroscopy [16, 20-22]. The parent zeolite shows a band centered at 210-220 nm, which corresponds to isolated tetrahedrally coordinated Ti species, together with a broad shoulder between 250-290 nm associated with highly coordinated (oligomeric) $\mathrm{Ti}[6,20-22]$. The presence of extra-framework $\mathrm{TiO}_{2}$, usually observed at 330 $\mathrm{nm}$ and upwards, is negligible [22]. Desilicated zeolites exhibit a similar UV-vis spectra with two broad contributions at 200-210 $\mathrm{nm}$ and 250-290 nm. The larger contribution of the latter peak (caused by the desilication treatment) is in agreement with the formation of penta-, hexa- or octa-coordinated Ti species formed by interaction with water molecules [14-17,20-22]. The presence of these species clearly indicates changes in the coordination for sites located outside the micropores due to their less confined and more hydrophilic environment, in good agreement with the desilication process [22]. The appearance of a shoulder centered at $330 \mathrm{~nm}$ suggests the presence of a certain amount of extra-framework titanium species due to the desilication treatment, as previously suggested by other authors $[14,16,17]$. Nevertheless, the presence of Ti tetracoordinated species after the desilication process is consistent with the preservation of some TS-1 entities on these 
samples besides the loss of crystallinity observed. Besides the selective nature of the desilication process, it is important to mention that these extra-framework titanium species are undesired due to their negative effect in oxidation reactions, i.e. these species initiate the decomposition of hydrogen peroxide.

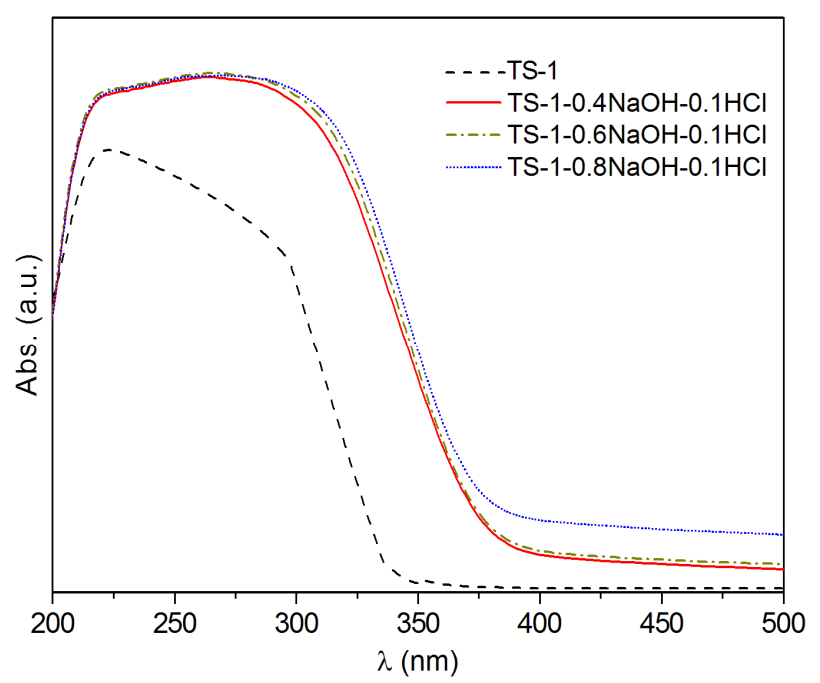

Fig. 4. UV-vis spectra of parent TS-1 and desilicated TS-1 zeolites.

The effect of mesoporosity on the catalytic properties of desilicated zeolites was evaluated in the oxidation of two organic molecules with very different sizes, cyclohexene and 1tert-butyl-1-cyclohexene, and using hydrogen peroxide as oxidant. Fig. 5 shows the correlation between the catalytic activity (conversion) and the BET surface area shown in Table 1. As it can be observed, TS-1, with a BET surface area of $c a .440 \mathrm{~m}^{2} / \mathrm{g}$ exhibits a large catalytic activity for cyclohexene with a total conversion after $2 \mathrm{~h}$ of around $62 \%$ (blank experiment for cyclohexene gives a conversion of 18\%). As expected, the catalytic activity of the same sample but using a large molecule, 1-tert-butyl-1-cyclohexene, is as low as $16 \%$ (blank experiment for 1-tert-butyl-1-cyclohexene gives a conversion of $6 \%$ ), confirming the diffusional limitations of the original TS-1 and its lack of intracrystalline mesoporosity, as observed by TEM (Fig. 1 left). However, the desilication treatment herein described drastically changes the catalytic performance of the samples. When TS-1 was chemically treated with $0.4 \mathrm{M} \mathrm{NaOH}$ its catalytic conversion for a small molecule 
such as cyclohexene decreases, which is consistent with the reduction in BET surface area, from 440 to $185 \mathrm{~m}^{2} \mathrm{~g}^{-1}$ (Table 1). But, its catalytic conversion increases, from $6 \%$ (for the original TS-1) up to 35\%, for 1-tert-butyl-1-cyclohexene. This behavior points out the detrimental effect of the chemical treatment, i.e. loss of titanium active sites, in oxidation reactions unless diffusional limitations are the rate determining step. An interesting result is observed for those samples chemically treated with a higher $\mathrm{NaOH}$ concentration. For both reactants, the catalytic activity increases with the BET surface area, this improvement being larger for a bulkier molecule as 1-tert-butyl-1-cyclohexene. These results confirm that the size exclusion effects are more evident for the original TS-1 sample, which imposes significant diffusional limitations due to its micropore structure. This behaviour is due to the absence of intra-particle mesoporosity in TS-1 sample, as evident by comparing the two micrographs shown in Fig. 1, as compared to the desilicated samples.

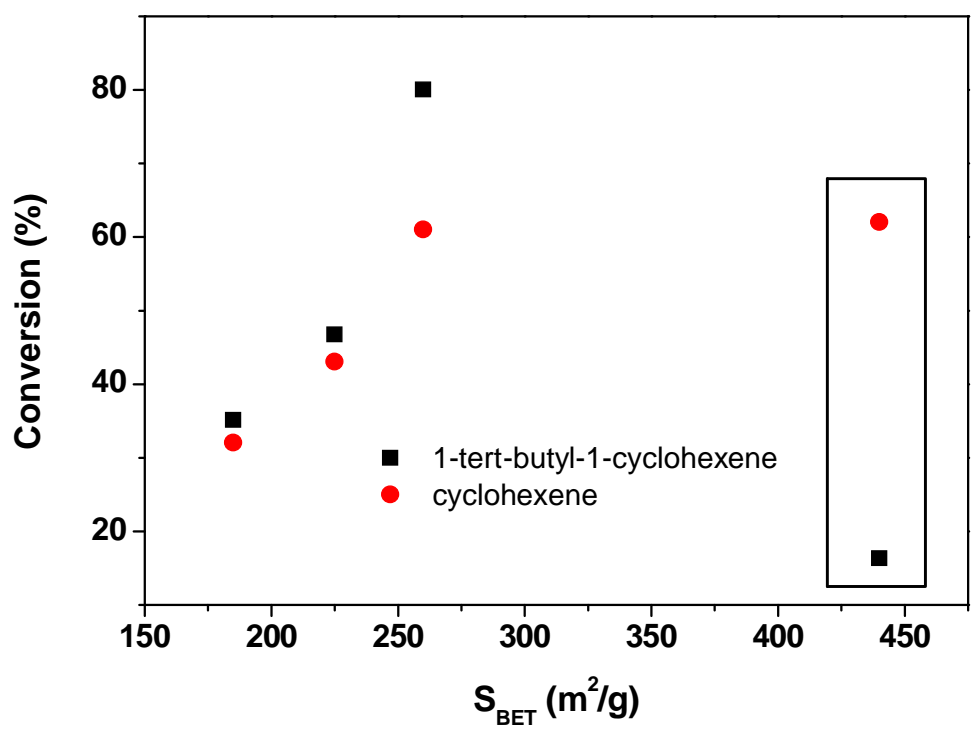

Fig. 5. Correlation between catalytic activity and BET surface area for the different desilicated TS-1 samples for the oxidation of cyclohexene and 1-tert-butyl-cyclohexene.

As expected, this intra-particle mesoporosity is the one contributing to a better diffusion of reactants to the active site. Furthermore, in the absence of intracrystalline diffusional 
limitations, the catalytic activity scales with the BET surface of the sample (see Fig. 5), up to a maxima in sample TS-1-0.8NaOH-HCl. This sample exhibits a catalytic activity for small molecules comparable to the original TS-1 (62\% vs. $61 \%$ conversion), whereas for bulky organic molecules a $400 \%$ increase in the catalytic activity is observed. These results open the possibility to get promising mesoporous zeolites for the epoxidation of bulky molecules unlocking the potential of this catalyst in industrial applications, despite the lower crystallinity of the as-synthesized mesoporous TS-1 zeolites as compared with other hierarchical TS-1 obtained by desilication with $\mathrm{NaOH}[6,8,14-17,22]$. Taking into account that the oxidation activity of Ti-containing zeolites is correlated to the framework titanium content, the catalytic performance of our hierarchical zeolites in oxidation reactions confirm that: i) a part of the tetrahedral Ti species remain in the structure of the zeolite TS-1 after the desilication treatment, ii) these Ti-active centers are accessible to bulky organic molecules, and iii) in the absence of kinetic restrictions, these centers remain the intrinsic catalytic activity of the Ti sites in the original TS-1.

\section{Conclusions}

In summary, this communication shows that the chemical treatment of TS- 1 zeolites with $\mathrm{NaOH}$ followed with $\mathrm{HCl}$ gives rise to the desilication of the zeolite and the formation of hierarchical structures containing well-developed mesopores. Under severe desilication conditions, X-ray amorphous materials are obtained. However, these Ti-rich materials show high BET surface area and excellent catalytic activity for the conversion of bulky molecules, such as butyl-1-cyclohexene, due to the presence of tetracoordinated Ti species and a very open porous structure which allows for an easy diffusion of reagents and products in and out of the active sites. 


\section{References}

[1] M. Taramasso, G. Perego, B. Notari, U.S. Patent 4,410,501 (1983).

[2] A. Corma, Chem. Rev. 97 (1997) 2373-2420.

[3] A. Corma, H. Garcia, Chem. Rev. 102 (2002) 3837-3892.

[4] I. Schmidt, A. Boisen, E. Gustavsson, K. Stahl, S. Pehrson, S. Dahl, A. Carlsson, C.J.H. Jacobsen, Chem. Mater. 13 (2001) 4416-4418.

[5] Y. Tao, H. Kanoh, K. Kaneko, J. Am. Chem. Soc. 125 (2003) 6044-6045.

[6] X. Wang, G. Li, W. Wang, C. Jin, Y. Chen, Microp. Mesop. Mater. 142 (2011) 494-502.

[7] F. Xiao, Y. Han, Y. Yu, X. Meng, M. Yang, S. Wu, J. Am. Chem. Soc. 124 (2002) 888889.

[8] M. Reichinger, W. Schmidt, M.W.E. van den Berg, A. Aerts, J.A. Martens, C.E.A Kirschhock, H. Gies, W. Grünert, J. Catal. 269 (2010) 367-375.

[9] S. van Donk, A. H. Janssen, J.H. Bitter, K.P. de Jong, Catal. Rev. 45 (2003) 297-319.

[10] J. C. Groen, J. C. Jansen, J. A. Moulijn, J. Pérez-Ramírez, J. Phys.Chem. B 108 (2004) 13062-13065.

[11] L. Zhao, J. Gao, C. Xu, B. Shen, Fuel Processing Technol. 92 (2011) 414-420.

[12] J.C. Groen, J.A. Moulijn, J. Pérez-Ramírez, J. Mater. Chem. 16 (2006) 2121-2131.

[13] J. Pérez-Ramírez, C.H. Christensen, K. Egeblad, C.H. Christensen, J.C. Groen, Chem. Soc. Rev. 37 (2008) 2530-2542.

[14] P.-Y. Chao, S.-T. Tsai, T.-C. Tsai, J. Mao, X.-W. Guo, Top. Catal. 52 (2009) 185-192.

[15] S.-T. Tsai, P.-Y. Chao, T.-C. Tsai, I. Wang, X. Liu, X.-W. Guo, Catal. Today 148 (2009) 174-178.

[16] J. Zhou, Z. Hua, X. Cui, Z. Ye, F. Cui, J. Shi, Chem. Commun. 46 (2010) 4994-4996.

[17] Y. Wang, M. Lin, A. Tuel, Microp. Mesop. Mater. 102 (2007) 80-85.

[18] M. Milina, S. Mitchell, Z.D. Trinidad, D. Verboekend, J. Pérez-Ramirez, Catal. Sci. Technol. 2 (2012) 759-766.

[19] X. Meng, D. Li, X. Yang, Y. Yu, S. Wu, Y. Han, Q. Yang, D. Jinag, F.-S. Xiao, J. Phys. Chem. B 107 (2003) 8972-8980.

[20] P. Ratnasamy, D. Srinivas, H. Knözinger, Adv. Catal. 48 (2004) 1-169.

[21] E. Duprey, P. Beaunier, M.-A. Springuel-Huet, F. Bozon-Verduraz, J. Fraissard, J.-M. Manoli, J.-M. Brégeault, J. Catal. 165 (1997) 22-32.

[22] D.P. Serrano, R. Sanz, P. Pizarro, I. Moreno, Appl. Catal. A: Gen 435-436 (2012) 32-42. 